\title{
Pneumococcal Nasopharyngeal Carriage in Young Healthy Children After Pneumococcal Conjugate Vaccine in Turkey
}

\author{
Ahmet Arvas1, Haluk Çokuğraş1, Emel Gür1, Nevriye Gönüllü2, Zeynep Taner², Hrisi Bahar Tokman² \\ ${ }^{1}$ Department of Paediatrics, İstanbul University Cerrahpaşa School of Medicine, İstanbul, Turkey \\ ${ }^{2}$ Department of Medical Microbiology, İstanbul University Cerrahpaşa School of Medicine, İstanbul, Turkey
}

\begin{abstract}
Aims: To determine the prevalence of nasopharyngeal carriage of Streptococcus pneumoniae in healthy children aged 0-6 years who were vaccinated with pneumococcal conjugate vaccine.
\end{abstract}

Methods: This cross-sectional study was conducted on 150 healthy Turkish children between 1 month and 6 years of age. Serotyping was performed and risk factors of carriage were evaluated.

Results: The overall carriage rate was $14 \%$. Vaccine type serotypes were determined in $17(12.6 \%)$ children who received full-dose PCV13 vaccine. The highest carriage rate was observed among children younger than 24 months (76.2\%). In multivariate analysis, respiratory infection in recent months, age, attendance at a day-care center and antibiotic usage were not statistically significant risk factors for carriage. Overall, $S$. pneumoniae strains were considered as penicillin susceptible and antimicrobial resistance was limited.

Conclusion: We observed a low rate of pneumococcal carriage in children after PCV13 implementation compared with that of children receiving PCV7. Although it was reduced, vaccine serotype colonization in PCV13-vaccinated children remains persistent.

Keywords: Children, nasopharyngeal carriage, pneumococcal conjugate vaccine, Streptococcus pneumoniae
Vaccine serotype (VT)-dependent invasive pneumococcal diseases (IPD) have been reduced significantly in immunised children and in non-vaccinated populations in countries where pneumococcal conjugate vaccine (PCV) has been introduced into the national immunisation program (NIP). PCV vaccine also reduces nasopharyngeal (NP) pneumococcal carriage and confers herd immunity on unvaccinated children and sensitive adults (1). Pneumococcal 7-valent vaccine (PCV7, serotype 4, 6B, 9V, $14,18 \mathrm{C}, 19 \mathrm{~F}$ and $23 \mathrm{~F}$ ) has been shown to be highly effective in reducing VT-dependent IPD and hospitalization. In addition, PCV7 has significantly decreased the prevalence of VT carriage
(2); however, there has also been a relative increase in the frequency of infections due to non-VT (NVT) such as 19A (3). The 13-valent PCV (PCV13), which comprises six additional serotypes $(1,3,5,6 \mathrm{~A}, 7 \mathrm{~F}, 19 \mathrm{~A})$ in addition to the serotypes in PCV7, has been widely used in immunisation programs since 2010. The efficacy of PCV13 against IPD varies between countries $(4,5)$.

The purpose of this study was to determine the carriage rate of NP Streptococcus pneumoniae in healthy children aged 0-6 years who were vaccinated with PCV and the serotype distribution and antimicrobial resistance of isolates.

\footnotetext{
Address for Correspondence: Dr. Ahmet Arvas, Department of Paediatrics, İstanbul University Cerrahpaşa School of Medicine, İstanbul, Turkey Phone: +90 21241430 00-21475 e-mail: aarvas@istanbul.edu.tr Received: 17 August $2016 \quad$ Accepted: 21 February 2017 • DOI: 10.4274/balkanmedj.2016.1256

Available at www.balkanmedicaljournal.org

Cite this article as:

Arvas A, Çokuğraş H, Gür E, Gönüllü N, Taner Z, Tokman HB. Pneumococcal Nasopharyngeal Carriage in Young Healthy Children After Pneumococcal Conjugate Vaccine in Turkey. Balkan Med J 2017;34:362-6

${ }^{\circ}$ Copyright 2017 by Trakya University Faculty of Medicine / The Balkan Medical Journal published by Galenos Publishing House.
} 


\section{MATERIALS AND METHODS}

This cross-sectional study involved 150 healthy Turkish children aged 0-6 years, attending the well-baby outpatient clinic in İstanbul University Cerrahpaşa School of Medicine, Department of Paediatrics between September and December 2014. PCV7 vaccine was implemented in the NIP in November 2008 as a 3+1 dose schedule at 2, 4, 6 and 12 months. It was replaced by PCV13 in April 2011, on the same schedule. The vaccination status of the children was determined as fully vaccinated (3 or 4 doses). A control group (unvaccinated children) was not included in this study, because the vaccination rate in Turkey is very high (98\%). Exclusion criteria for enrolment were acute (e.g., respiratory infection) and chronic diseases. Information was obtained from parents using a questionnaire on demographics, household properties, risk factors for carriage, history of upper respiratory tract infections and antimicrobial use.

NP specimens were transferred to the microbiology laboratory in liquid Amie's transport medium (Copan Diagnostics, Brescia, It.). The swabs were streaked on 5\% sheep's blood agar chocolate agar plates. Gram-positive diplococci appearance in gram-stained smears were assessed as streptococci. The identification of $S$. pneumoniae was confirmed via optochin susceptibility and bile solubility testing. The serotypes of pneumococcal isolates including PCV13 serotypes and nonPCV13 serotypes (7A, 7B, 7C, 19A, 19B, 19C) were detected using a commercial latex agglutination kit (Pneumotest-Latex kit; Statens Serum Institude, Denmark). Confirmation and factor typing were performed by the capsular reaction test (Quellung reaction) using specific antisera (SSI). Antimicrobial susceptibility testing of $S$. pneumoniae strains was performed according to standard procedures as previously described. The minimum inhibitory concentration values of antibiotics were determined via the E test (Etest, Biomerieux, Paris, France). The susceptibility results were evaluated according to the European Committee on Antimicrobial Susceptibility Testing (EUCAST 2015) guidelines (6-8).

The study was approved by the Ethics Committee of İstanbul University Cerrahpaşa School of Medicine (A-50). Written informed consent was provided by the parents of each enrolled child. This study was funded by the Turkish Paediatric Association (grant number: 05).

\section{Statistical analysis}

The software program SPSS 22.0 was used for statistical analyses. Frequencies for categorical variables were evaluated by percentiles. Continuous variables, if they fit with the normal distribution were expressed as means \pm standard deviation; if they did not fit, they were expressed as medians (minimum- maximum). The relationship between NP carriage and age was evaluated by chi-square test. The independent variables affecting NP carriage were identified by univariate analysis and were evaluated with odds ratios and 95\% confidence intervals (CI). Significant variables therein were subjected to multivariate logistic regression analysis.

\section{RESULTS}

The mean age of the children was $23.7 \pm 14.3$ months; $49 \%$ of the participants were males. More than $90 \%$ of the children had received full doses of PCV13 (3 or $3+1)$. Descriptive characteristics of the children are presented in Table 1.

The overall NP pneumococcal carriage rate was 14\% (21/150). The NP carriage rate of children vaccinated with PCV7 $(3+1)$ was $21.4 \%(3 / 14)$, whereas it was $11.7 \%(6 / 51)$ in children

TABLE 1. Characteristics of the study population

\begin{tabular}{|c|c|c|}
\hline \multicolumn{3}{|l|}{ Characteristics $(n=150)$} \\
\hline Age (months) & $23.7( \pm 1.42)^{*}$ & \\
\hline Breast feeding (months) & $12.5( \pm 7.8)$ & \\
\hline Siblings & $1(0-4) * *$ & \\
\hline \multirow[t]{2}{*}{ The number of people living at home } & $4(3-10) * *$ & \\
\hline & $\mathrm{n}$ & $\%$ \\
\hline \multicolumn{3}{|l|}{ Sex } \\
\hline Male & 74 & 49.3 \\
\hline Female & 76 & 50.7 \\
\hline \multicolumn{3}{|l|}{ Vaccination status } \\
\hline PCV7 (3 doses) & 2 & 1.3 \\
\hline PCV7 (3+1 doses) & 14 & 9.3 \\
\hline PCV13 (3 doses) & 51 & 34.0 \\
\hline PCV13 (3+1 doses) & 83 & 55.3 \\
\hline \multicolumn{3}{|l|}{ Respiratory infection } \\
\hline Last month & 35 & 23.3 \\
\hline Last three months & 41 & 27.4 \\
\hline \multicolumn{3}{|l|}{ Antibiotic usage } \\
\hline Last month & 34 & 22.7 \\
\hline \multicolumn{3}{|l|}{ Attendance at day care centres } \\
\hline No & 94 & 62.7 \\
\hline Himself/herself & 6 & 4.0 \\
\hline Sibling & 43 & 28.7 \\
\hline Himself/herself + sibling & 7 & 4.7 \\
\hline \multicolumn{3}{|l|}{ Education of mother } \\
\hline$\leq$ Primary school & 32 & 21.3 \\
\hline \multicolumn{3}{|l|}{ Education of father } \\
\hline$\leq$ Primary school & 36 & 24.0 \\
\hline \multicolumn{3}{|l|}{ Exposure to smoke at home } \\
\hline Yes & 55 & 36.7 \\
\hline
\end{tabular}


vaccinated with PCV13 (3 doses) and 14.4\% (12/83 children) in those with PCV13 $(3+1)$. The highest carriage rate of $76.2 \%$ (16/21 children) was observed among children younger than 24 months with no significant differences between age groups $(\mathrm{p}=0.495)$ (Table 2$)$.

Twenty carriers had more than one serotype. Of the 81 isolates, nine different serotypes were identified. Thirty-six isolates were PCV13 VTs, whereas 44 isolates were non-VT isolates. Three isolates had serotypes included in PCV7, whereas nine isolates did not.

The risk factors of having siblings, people living at home, day care attendance and smoking in the home were found not to affect NP carriage $(\mathrm{p}=0.572, \mathrm{p}=0.900, \mathrm{p}=0.145$ and $\mathrm{p}=0.406$ respectively), whereas NP carriage was associated with a respiratory infection in the last 3 months $(\mathrm{p}=0.048$ ). Univariate and multivariate logistic regression analysis results are shown in Table 3. We did not detect any risk factors in the multivariate analysis.

Serotypes 19A, 19B, 19C and 19F were detected in three children vaccinated with PCV7 ( $3+1$ doses). Serotypes 7A, 7B, 7C and 7F were detected in two children vaccinated with PCV13 (3 doses), whereas serotypes 19A, 19B, 19C and 19F were detected in four children vaccinated with the same doses. Serotypes 7A, 7B, 7C and $7 \mathrm{~F}$ were detected in two children vaccinated with PCV13 (3+1 doses), whereas serotypes 19A, 19B, 19C and 19F were in nine children vaccinated with the same doses (Figure 1).

Penicillin susceptibility was identified in $85.7 \%$ of isolated strains. All isolates $(100 \%)$ were vancomycin susceptible, whereas $85.7 \%$ of them were susceptible to cefaclore, $52.3 \%$ were susceptible to erythromycin, $47.6 \%$ were susceptible to clindamycin and $42.8 \%$ were susceptible to trimethoprim/ sulphamethoxazole.

TABLE 2. Relationship between pneumococcal carriage and age in children

\begin{tabular}{lccc}
\hline Age (months) & $\begin{array}{c}\text { S. pneumoniae }(+) \\
\mathrm{n}(\%)\end{array}$ & $\begin{array}{c}\text { S. pneumoniae }(-) \\
\mathrm{n}(\%)\end{array}$ & $\mathrm{p}^{*}$ \\
\hline $0-24$ months & $16(76.2)$ & $85(65.9)$ & \\
$25-60$ months & $5(23.8)$ & $44(34.1)$ & 0.495 \\
\hline *chi-square yates & & &
\end{tabular}

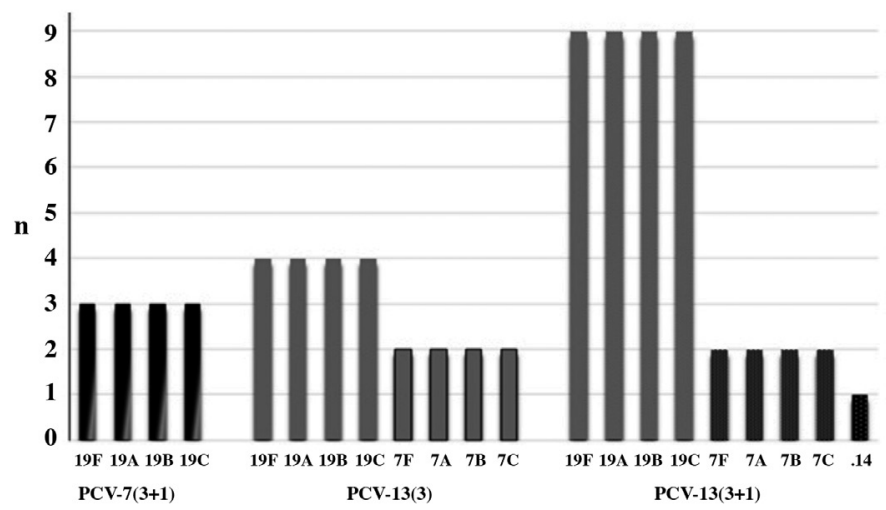

FIG. 1. Serotype distribution of the nasopharyngeal carriage $(n=21)$ according to the vaccination status PCV: pneumococcal conjugate vaccine

\section{DISCUSSION}

In this study, the overall rate of NP carriage was $14 \%$ among full-dose vaccinated children. NP pneumococcal colonization varies according to region and community. In developing countries, the rate of NP carriage in children under 5 years of age was found to be $8 \%-75 \%$, and in developed countries, it was $13 \%-77 \%$ before the introduction of PCV into NP (9). Several surveys have documented the effect of PCV on NP carriage with conflicting results (10-12). The main reason for the unchanged prevalence of NP carriage is serotype replacement of non-vaccine types $(13,14)$. After the introduction of PCV7, the incidence of pneumococcal disease and NP carriage due to NVTs, most notably for serotype 19A, increased incrementally. Dunais et al. (15). observed that the rate of NP carriage decreased significantly after PCV7 introduction; on the other hand, a further significant decrease was not seen following the implementation of PCV13, and the seroprevalence of 19A in NP colonization was persistent. Lee et al. (16) reported stable colonization rates after PCV13, with significant increases in non-PCV13 serotypes.

In this study, NP carriage prevalence was found to be higher in children attending a day-care centre. In a study carried out

TABLE 3. Examination of the risk cases for NP carriage in univariate and multivariate analyses

\begin{tabular}{|c|c|c|c|c|c|c|}
\hline \multirow{2}{*}{ Characteristics } & \multicolumn{3}{|c|}{ Univariate analysis } & \multicolumn{3}{|c|}{ Multivariate analysis } \\
\hline & OR & $95 \% \mathrm{CI}^{*}$ & $\mathrm{p}$ & $\operatorname{Exp}(B)$ & $95 \% \mathrm{CI}$ & $\mathrm{p}$ \\
\hline Age ( $>24$ months) & 0.604 & $0.207-1.757$ & 0.285 & 1.844 & $0.608-5.593$ & 0.618 \\
\hline Infections on the last 3 months (no) & 4.117 & $0.914-18.536$ & 0.048 & 0.317 & $0.033-3.026$ & 0.378 \\
\hline Day care attendance (no) & 0.487 & $0.192-1.234$ & 0.145 & 2.638 & $0.765-9.098$ & 0.545 \\
\hline Antibiotic usage (no) & 3.134 & $0.692-14.198$ & 0.512 & 0.742 & $0.075-7.323$ & 0.550 \\
\hline Sibling (no) & 0.844 & $0.318-2.237$ & 0.572 & 0.729 & $0.201-2.646$ & 0.576 \\
\hline Constant & & & & 0.110 & & \\
\hline
\end{tabular}


in Hungary, the NP carriage rate among unvaccinated children attending a day-care centre was also found to be higher (13). Özdemir et al. (17) reported that the NP carriage rate in healthy children was not influenced by PCV7 (21.9\%). We suggest that the reason for the lower NP carriage rate in our study compared with the study mentioned above is the that PCV13 includes more serotypes than PCV7. Soysal et al. (18) reported 8\%, 7\% and 5\% NP carriage rates among age groups of 0-24 months, 25-60 months and >60 months, respectively. Sixty percent of children in this study were not vaccinated with any PCV, whereas $30 \%$ of children received the full schedule (4 doses) of either PCV7 or PCV13.

We did not observe any association between NP carriage and gender, whereas some studies have reported that the male gender is a risk factor (15). The NP carriage rate in our study was higher in children who had a respiratory tract infection, similar to several other studies $(11,17)$. We could not observe any positive effects of using antibiotics on carriage. A study in Italy reported that the use of antibiotics in the previous 3 months was associated with lower NP carriage prevalence, whereas having siblings, attending a day-care centre and having had a respiratory tract infection in the last 3 months were associated with higher NP carriage prevalence (12).

In the present study, the NP carriage rate in children vaccinated with PCV7 ( 3 or $3+1$ doses) was found to be higher than that in children vaccinated with PCV13 (18.7\% vs 13.4\%). The reason for more effective prevention by PCV13 than PCV7 is that it contains more serotypes, similar to the findings of other studies $(12,16,19)$.

We observed that 3 children vaccinated with PCV7 had the $19 \mathrm{~F}$ serotype, 4 children vaccinated with PCV13 (3/3+1 doses) had 7F and 13 cases vaccinated with PCV13 had 19A and 19F serotypes. A study by Grivea et al. (19) determined that $15.8 \%$ of the children attending a day-care centre had the 19A serotype 6 months after PCV13 vaccination. In addition, the authors reported that, although there was no 19F serotype carriage in the first year of PCV7 vaccination, after the first year, the prevalence of serotype $19 \mathrm{~F}$ was $2.2 \%$. NP colonization containing VTs is also noteworthy in some studies, and this situation can be explained with "immune-hyporesponsiveness to the colonizing serotype following PCV" $(16,20)$.

In our study, pneumococcal isolates in NP colonization were $85 \%$ penicillin sensitive. In a study by Zuccotti et al. (12) in Italy, it was observed that $69 \%$ of the pneumococcus isolates from NP carriers after PCV-13 vaccination were penicillin sensitive, $29 \%$ were intermediate sensitive and the most frequently isolated resistant serotype was $19 \mathrm{~F}$.

Studies similar to ours reported that, following PCV introduction, a significant reduction was observed in penicillin- resistant colonizing $S$. pneumoniae isolates due to VTs. Several studies observed that the 19A serotype was the most resistant $(10,13,15)$.

There are some limitations to this study. First, we had no prePCV period cohort with which to compare our study population. Second, this study is a cross-sectional study. Due to the lack of a long-term surveillance study of the same cases, it will not be possible to determine whether the NP carriage frequency and serotypes change in the future. Third, the present study has a relatively small sample size and does not include children older than 6 years. Therefore, it is not possible to determine the frequency of NP pneumococcal carriage in all children.

In conclusion, this study determined the lower NP carriage prevalence after PCV-13 implementation compared with previous studies before or after PCV-7 implementation. This study also determined that, in the early childhood years, only having a respiratory tract infection has a positive effect on NP colonization. There are some VTs of NP carriage with low frequency. In Turkey, where the coverage rate of vaccination in children is quite high, studies including a large sample size and a broader age range would be more useful in determining the actual long-term NP carriage status, which is very important in IPD and herd immunity.

Financial Disclosure: This study was funded by the Turkish Paediatric Association (grant number: 05).

Conflict of Interest: No conflict of interest was declared by the authors

\section{REFERENCES}

1. Centers for Disease Control and Prevention (CDC) Direct and indirect effects of routine vaccination of children with 7-valent pneumococcal conjugate vaccine on incidence of invasive pneumococcal disease United States, 1998-2003. MMWR Morb Mortal Wkly Rep 2005;54:893-7.

2. Fitzwater SP, Chandran A, Santosham M, Johnson HL. The worldwide impact of the seven-valent pneumococcal conjugate vaccine. Pediatr Infect Dis J 2012;31:501-8.

3. Chapman KE, Wilson D, Gorton R. Serotype dynamics of invasive pneumococcal disease post PCV-7 and pre PCV-13 introduction in North East England. Epidemiol Infect 2013;141:344-52.

4. Hackel M, Lascols C, Bouchillon S, Hilton B, Morgenstern D, Purdy J. Serotype prevalence and antibiotic resistance in Streptococcus pneumoniae clinical isolates among global population. Vaccine 2013;31:4881-7.

5. Ceyhan M, Gurler N, Yaman A, Ozturk C, Oksuz L, Ozkan S, et al. Sertypes of Streptococcus pneumoniae isolates from children with invasive pneumococcal disease in Turkey: baseline evaluation of the introduction of the pneumococcal conjugate vaccine nationwide. Clin Vaccine Immunol 2011;18:1028-30.

6. Slotved HC, Kaltoft M, Skovsted IC, Kerrn MB, Espersen F. Simple rapid latex agglutination test for serotyping of pneumococci (Pneumotest-Latex). J Clin Microbiol 2004;42:2518-22. 
7. Shutt CK, Samore M, Carroll CK. Comparison of the denka seiken slide agglutination method to the quellung test for serogrouping of Streptococcus pneumoniae isolates. J Clin Microbiol 2004;42:1274-6.

8. European Committee on Antimicrobial Susceptibility Testing. Breakpoint tables for interpretation of MICs and zone diameters. Version 5.0, 2015. Available from: http://www.eucast.org (7 July 2015, date last accessed).

9. Nzenze SA, Shiri T, Nunes MC, Klugman KP, Kahn K, Twine R, et al. Temporal association of infant immunization with pneumococcal conjugate vaccine on the ecology of Streptococcus pneumoniae, Haemophilus inluenzae and Staphylococcus aureus nasopharyngeal colonization in a rural South African community. Vaccine 2014;32:5520-30.

10. Daana M, Rahav G, Hamdan A, Thalji A, Jaar F, Abdeen Z, et al. Measuring the effects of pneumococcal conjugate vaccine (PCV7) on Streptococcus pneumoniae carriage and antibiotic resistance: The Palestinian-Israeli Collaborative Research (PICR). Vaccine 2015;33:1023-6.

11. Dagan R, Patterson S, Juergens C, Greenberg D, Givon-Lavi N, Porat $\mathrm{N}$, et al. Comparative immunogenicity and efficacy of 13 -valent and 7 -valent pneumococcal conjugate vaccines in reducing nasopharyngeal colonization: a randomized double-blind trial. Clin Infect Dis 2013;57:95262.

12. Zuccotti G, Mameli C, Daprai L, Garlaschi ML, Dilillo D, Bedogni G, et al. Serotype distribution and antimicrobial susceptibilities of nasopharyngeal isolates of Streptococcus pneumoniae from healthy children in the 13-valent pneumococcal conjugate vaccine era. Vaccine 2014;32:527-34.

13. Tothpal A, Kardos S, Laub K, Nagy K, Tirczka T, van der Linden M, et al. Radical serotype rearrangement of carrier pneumococci in the first 3 years after intensive vaccination started in Hungary. Eur J Pediatr 2015;174:37381.
14. Scott JR, Millar EV, Lipsitch M, Moulton LH, Weatherholtz R, Perilla MJ, et al. Impact of more than a decade of pneumococcal conjugate vaccine use on carriage and invasive potential in Native American communities. J Infect Dis 2012;205:280-8.

15. Dunais B, Bruno P, Touboul P, Degand N, Sakarovitch C, Fontas E, et al. Impact of the 13-valent pneumococcal conjugate vaccine on nasopharyngeal carriage of Streptococcus pneumoniae among children attending group daycare in southeastern France. Pediatr Infect Dis J 2015;34:286-8.

16. Lee GM, Kleinman K, Pelton SI, Hanage W, Huang SS, Lakoma M, et al. Impact of the 13-valent pneumococcal conjugate vaccine on Streptococcus pneumoniae carriage in young children in Massachusettes. J Pediatr Infect Dis Soc 2014;3:23-32.

17. Özdemir H, Çiftçi E, Durmaz R, Güriz H, Aysev AD, Karbuz A, et al. Risk factors for nosopharyngeal carriage of Streptococcus pneumoniae in healthy Turkish children after the addition of heptavalent pneumococcal conjugate vaccine (PCV7) to the national vaccine schedule. Turk J Pediatr 2013;55:575-83.

18. Soysal A, Karabağ-Yılmaz E, Kepenekli E, Karaaslan A, Cagan E, Atıcı $\mathrm{S}$, et al. The impact of a pneumococcal vaccination program on the nasopharyngeal carriage, serotype distribution and antimicrobial resistance of Streptococcus pneumoniae among healthy children in Turkey. Vaccine 2016;34:3894-900.

19. Grivea IN, Priftis KN, Giotas A, Kotzia D, Tsantouli AG, Douros K, et al. Dynamics of pneumococcal carriage among day-care center attendees during the transition form the 7-valent to the higher-valent pneumoccal conjugate vaccines in Greece. Vaccine 2014;32:6513-20.

20. Rivera-Olivero IA, del Nogal B, Fuentes M, Cortez R, Bogaert D, Hermans $\mathrm{PW}$, et al. Immunogenicity of a 7-valent pneumococcal conjugate vaccine (PCV7) and impact on carriage in Venezuelan children at risk of invasive pneumococcal diseases. Vaccine 2014;32:4006-11. 